\title{
Early Adolescent Depressive Symptoms: Prediction from Clique Isolation, Loneliness, and Perceived Social Acceptance
}

\author{
Miranda Witvliet - Mara Brendgen • \\ Pol A. C. van Lier • Hans M. Koot • Frank Vitaro
}

Published online: 25 May 2010

(C) The Author(s) 2010. This article is published with open access at Springerlink.com

\begin{abstract}
This study examined whether clique isolation predicted an increase in depressive symptoms and whether this association was mediated by loneliness and perceived social acceptance in 310 children followed from age 1114 years. Clique isolation was identified through social network analysis, whereas depressive symptoms, loneliness, and perceived social acceptance were assessed using self ratings. While accounting for initial levels of depressive symptoms, peer rejection, and friendlessness at age 11 years, a high probability of being isolated from cliques from age 11 to 13 years predicted depressive symptoms at age 14 years. The link between clique isolation and depressive symptoms was mediated by loneliness, but not by perceived social acceptance. No sex differences were found in the associations between clique isolation and depressive symptoms. These results suggest that clique isolation is a social risk factor for the escalation of
\end{abstract}

\footnotetext{
M. Witvliet

Department of Clinical Psychology and Developmental Psychology,

VU University Amsterdam,

Amsterdam, the Netherlands

M. Brendgen

Department of Psychology, Université du Québec à Montréal,

Montreal, Canada

P. A. C. van Lier $\cdot$ H. M. Koot $(\varangle)$

Department of Developmental Psychology,

VU University Amsterdam,

van der Boechorststraat 1,

1081 BT Amsterdam, the Netherlands

e-mail: jm.koot@psy.vu.nl

F. Vitaro

GRIP, University of Montreal,

Montreal, Canada
}

depressive symptoms in early adolescence. Implications for research and prevention are discussed.

Keywords Depressive symptoms · Loneliness .

Perceived social acceptance $\cdot$ Cliques $\cdot$ Peer Relations

Symptoms of depression increase sharply in adolescence and are common during this age period (Birmaher et al. 1996; Hankin et al. 1998; Petersen et al. 1993). Furthermore, symptoms of depression may develop into more serious psychiatric problems including major depressive disorder (Pine et al. 1999). Depression is believed to occur within an interpersonal context, and problematic interpersonal relations are often identified as predictors of depression (Joiner 1997). According to the interpersonal theory of depression (Coyne 1976), an escalating cycle is established within depressed persons' social relationships. That is, the problematic social relationships of depressed persons are hypothesized to have an additive effect on the development of their depressive symptoms. This interpersonal perspective of depression, in which problematic peer relations are thus regarded as risk factors for an escalation in depressive symptoms, is frequently included in contemporary theories of depression in childhood and adolescence (Rudolph et al. 2008).

In line with the notion that childhood and adolescent depression amplifies within an interpersonal context, empirical evidence has been found for an association between problematic peer relations and depressive symptoms among children and adolescents. That is, peer rejection in childhood and adolescence has been shown to be associated with a heightened risk for depressed mood (Boivin et al. 1995, 1994; Brendgen et al. 2005; Ladd and Troop-Gordon 2003). Besides peer rejection, there are other peer relational risk variables that play a role in the 
development of depressive symptoms. For instance, children's dyadic friendships, which can be distinguished from children's sociometric status (Parker and Asher 1993), are related to feelings of depression. That is, children and adolescents who are friendless have been shown to be more depressed than others (Pedersen et al. 2007). It is important to note, though, that children's dyadic friendships are often nested within larger social networks, for instance in cliques. Cliques can be defined as self-selective, friendship-based groups of peers who are highly interconnected (Shrum and Cheek 1987; Urberg et al. 1995; Kindermann 2007). As we will argue, being isolated from cliques, which reflects not being a member of friendship groups of mainstream peers, may uniquely predict depressive symptoms.

Because cliques are friendship-based groups, cliques can be distinguished from sociometric status. That is, sociometric status reflects how much a child is liked or disliked by his/her peers rather than the number of actual friends a child has (Parker and Asher 1993). A child with a negative peer reputation may still affiliate in cliques (Bagwell et al. 2000). And in contrast, children who are isolated from cliques may still be liked by their peers (Wentzel and Caldwell 1997). Moreover, cliques provide children with resources, such as a sense of collective participation and group support, which dyadic friendships often cannot offer (Rubin 1980). Indeed, cliques have specific structural characteristics, such as transitivity, stratification, and cohesiveness that move beyond characteristics of dyadic friendships (Adler and Adler 1998; Wasserman and Faust 1994). This conceptual distinction between dyadic friendships and cliques has also received empirical support. For instance, Wentzel and Caldwell (1997) showed that children's reciprocated friendships and their clique membership were differently related to their academic achievement in middle school. Consequently, children who are isolated from cliques are deprived of different assets (i.e., group support, and a sense of belongingness) than children who are not part of dyadic friendships or who are rejected by peers.

As said, being isolated from cliques may deny children positive group experiences and may therefore put children at risk for intensifying their depressive symptoms (Brown and Lohr 1987; Hoza et al. 2000). This may particularly be the case in late childhood and early adolescence. While close and intimate friendships may become more significant for children's development during adolescence (Sullivan 1953), achieving group identity and group acceptance is believed to be a central developmental task in late childhood and early adolescence (Parker and Gottman 1989; Buhrmester 1990; Buhrmester and Furman 1987). In line with this notion, initial evidence indicates that cliques isolates show more depressive symptoms than members of clique. That is, Henrich et al. (2000) identified clique members and clique isolates based on patterns of reciprocated close friendship nominations and found that sixth and seventh grade clique isolates show more teacher rated internalizing problems - including more depressed mood - than clique members. Nonetheless, the role of clique isolation in the development of children's depressive symptoms is uncertain. In addition, it is unknown whether clique isolation is associated with depressive symptoms above and beyond other forms of peer relations. Indeed, although clique isolation, peer rejection and friendlessness are regarded as distinct peer relation problems, children who are isolated from cliques often have fewer reciprocated friendships and are less accepted by their peers than clique members (Wentzel and Caldwell 1997). The first objective of the present study was therefore to investigate whether being isolated from cliques from age 11 to 13 years predicted an increase in depressive symptoms at age 14 years, while controlling for other problems in the peer relations domain.

\section{Loneliness and Perceived Social Acceptance as Potential Mediators of the Link between Clique Isolation and Depressive Symptoms}

It is uncertain whether there exists a direct effect of problematic peer relations on depressive symptoms. Rather, it has been theorized that poor experiences with peers likely evoke emotional responses such as loneliness and cognitive reactions including poor self-esteem. These cognitiveemotional constructs are expected to underlie depressive symptoms (Beck 1987; Joiner 1997; Joiner et al. 2002; Kovacs and Beck 1978). For instance, Rubin et al. (2003) argue that socially withdrawn children attribute their negative relationships with peers to internal causes and may as a consequence experience feelings of loneliness and depression. In addition, feelings of loneliness can be expected to predict an increase in depressive symptoms (Brage and Meredith 1994; Joiner 1997). Findings from recent studies indeed provide support for a mediating model by which children's poor peer experiences have an indirect effect on depressive symptoms via feelings of loneliness. For instance, Fontaine et al. (2009) showed that feelings of loneliness mediated the relationship between low social preference in childhood and depressed symptoms in adolescence. Also, Boivin et al. (1995) found that loneliness had a mediating effect on the relationship between children's social conditions and depressive outcomes. Although not directly studied, it can be theorized that loneliness follows from being isolated from cliques. For instance, Hoza et al. (2000) argued for the existence of multiple types of loneliness. That is, feelings of loneliness can arise from deficits in dyadic relationships, but also from deficits in the peer group, which Hoza and colleagues describe as 'network loneliness'. Hence, loneliness can be argued to be the internalized emotional experience of not 
only problems with dyadic friendships, but also of being isolated from cliques.

There is also theoretical and empirical support for the mediating role of poor self-esteem, particularly children's selfesteem in the social domain (perceived social acceptance; Harter 1982). For instance, according to vulnerability-stress models of depression (Abramson et al. 1989; Beck 1987; Kovacs and Beck 1978), negative stressful life events, such as poor experiences with peers, can be internalized into maladaptive self-schemata. These negative cognitions about the self may, subsequently, cause increases in depressive symptoms. Indeed, the association between low self-esteem and depressive symptoms in adolescence has been demonstrated in numerous studies (e.g., MacPhee and Andrews 2006; Orth et al. 2008; Pelkonen et al. 2008). Mead (1934) suggested that the self-concept of children and adolescents is largely formed through peer interactions. Harter et al. (1996) found support for a 'looking glass self-orientation' among adolescents: adolescents like themselves based on the approval of peers. In a similar way, according to Cole's (1991) competency-based model of depression, a child's sense of self-competence is largely based on the feedback he/ she receives about various areas of life, including the social domain. Low levels of self-perceived competence are, in turn, believed to predict the development of depression. In line with this notion, it can be proposed that negative group experiences, such as the experience of being isolated from cliques, may negatively impact children's self-esteem and in particular children's perceived social acceptance. Therefore, as a second objective of the present study, we investigated whether being isolated from cliques from age 11 to 13 years was associated with an increase in depressive symptoms via loneliness and low perceived social acceptance.

\section{The Child's Sex and the Link between Clique Isolation and Depressive Symptoms}

Depressive symptoms have been found to be more prevalent among adolescent girls than boys (AllgoodMerten et al. 1990; Brage and Meredith 1994). However, it is unknown whether this sex difference in prevalence of depressive symptoms also translates into sex-specific links between clique isolation and depressive symptoms. Prior findings indicate that girls are more often members of a clique and place greater importance on clique membership than boys (Cohen 1977; Urberg et al. 1995). Therefore, depressive symptoms may be observed especially among girls who are outside cliques. However, the link between depressive symptoms and problematic peer relations, such as peer rejection and friendlessness, has often been reported to be sex-invariant (Ladd and Troop-Gordon 2003; La Greca and Moore Harrison 2005; Pedersen et al. 2007). Because these studies have not focused on clique isolation specifically, it is uncertain whether the link between clique isolation and depressive symptoms is different for boys and girls.

\section{The Present Study}

In sum, the first objective of the present study was to investigate whether clique isolation is a social risk factor for an escalation in depressive symptoms in early adolescence. The second objective was to study whether the association between clique isolation and depressive symptoms is mediated by cognitive-emotional constructs. We identified cliques in classrooms with social network analysis based on children's friendship nominations. We used this method because we were interested in identifying children who were not part of friendship-based groups (Kindermann and Gest 2009). In particular children who are isolated from such friendship groups are expected to be denied of the possibility to attain positive group experiences including a sense of belongingness and support from other clique members, resulting in cognitive-emotional responses such as feelings of loneliness and low perceived social acceptance, and subsequent depressive symptoms. Although there are other methods available for the identification of cliques (e.g., by asking children to report about a group of friends or by own observations from the researcher), social network analysis is often used by developmental psychologists and sociologists to detect cliques and to identify clique isolates (see e.g., Ennett and Bauman 1994; Espelage et al. 2003; Urberg et al. 1995). We hypothesize that clique isolation at age 11 to 13 years would predict self-reported depressive symptoms at age 14 years, even if initial levels of depressive symptoms at age 11 years and other problems in the peer relations domain (i.e., peer rejection and friendlessness at age 11) would be controlled for. We also hypothesize that the association between clique isolation and depressive symptoms would be mediated by loneliness and perceived social acceptance. In each of these objectives, we explored whether the results were different for boys and girls. We hypothesize that boys would be more often isolated from cliques than girls. Moreover, because sex-specific links between clique isolation and internalizing problems are not previously studied, we have no specific expectations regarding sex differences in the association between clique isolation and depressive symptoms.

\section{Method}

Participants

Participants included 310 children (53\% boys) from a small community in northwestern Quebec, Canada. These children 
participated in a longitudinal study that included 381 participants at the first assessment point relevant to this study (grade 5). Participants attended five elementary schools up to grade 6 , after which they transferred to one large high school. The majority of children $(>90 \%)$ had a French Canadian background. In each year of data-collection, all French speaking children in the community were targeted for participation in the study. At least $90 \%$ of children from the targeted classrooms participated in the study in each year. The average age of the included children was 11.10 years $(S D=$ 0.36 ) in grade 5 (i.e., the initial time point of the present study). Participants were followed over 3 years, until age 14 (the end of grade 8). Participant's socioeconomic status, measured with the Blishen et al. (1987) occupational prestige scale $(M=40.16, S D=13.59)$, was in accordance with a representative sample throughout the Province of Quebec, Canada $(M=43.99, S D=13.00)$. Seventy-four percent of children lived in intact families, $10 \%$ lived in blended family households, $13 \%$ in single parent families, and $3 \%$ lived in other family configurations.

To be included in the present study, children needed to have information on at least two data points on clique isolation measured from age 11-13 years and depressive symptoms measured at age 14 years. In total 310 children were included in the study sample by virtue of this criterion. Of these 310 children, 289 children (93\%) had information on at least three out of four data points and 269 children had complete data on these study variables $(87 \%)$. Attrition was due to no availability of parental permission, moving out of the school district, or absence on the day of data collection. For children who had missing data on any of the four time points, missing values were estimated by using a procedure known as Full Information Maximum Likelihood (FIML). The assumption underlying FIML is that data are missing at random (MAR). The children included in the final sample were compared with children who did not meet the study criteria on the study variables in grade 5. No differences were found in depressive symptoms $(F(1,396)=3.68, p>0.05)$, perceived social acceptance $(F$ $(1,353)=3.14, p>0.05)$, loneliness $(F(1,402)=2.88, p>$ $0.05)$, clique membership status $\left(\chi^{2}(1, N=284)=1.81, p>\right.$ $0.05)$, friendlessness $\left(\chi^{2}(1, N=270)=0.03, p>0.05\right)$, rejection $\left(\chi^{2}(1, N=381)=1.97, p>0.05\right)$, sex $\left(\chi^{2}(1, N=\right.$ $484)=0.47, p>0.05$ ). However, children who were included in the final sample had a higher parental occupational prestige than children outside the sample $(F(1,293)=4.31$, $p=0.04$ ).

\section{Measures}

Depressive Symptoms The Children's Depression Inventory (CDI; Kovacs 1992) was used to obtain children's selfreported depressive symptoms at age 11 and age 14 years.
The CDI has been validated using normative and clinicreferred samples and has been found to be reliable (Fundulis et al. 1991). Using 26 out of the 27 items of the CDI, children indicated for each item whether they had an absence of symptoms (0), had mild symptoms (1), or definite symptoms (2). The suicidal ideation item was left out of the questionnaire due to concerns of the school administration. The severity of a given child's depressive symptoms was measured by summing his or her item scores $(\alpha=0.85$ at age 11 years and $\alpha=0.86$ at age 14 years).

Perceived Social Acceptance The Self-Perception Profile for Children (SPPC; Harter 1985) was completed by children each year from age 11 to 13 years to assess perceived social acceptance. The SPPC measures children's self perception across domains of scholastic, athletic, physical, behavioral, and social competencies, as well as global self-worth. The SPPC has been found to be a reliable and valid measure of children's self perception (Boivin et al. 1992; Harter 1985). We measured perceived social acceptance by using the items from the social acceptance domain. However, we omitted the item 'number of friends' to avoid too much overlap with the other constructs measured in this study. The items are scored from 1 to 4 , with higher scores reflecting more positive self-perceptions. Cronbach's alpha for perceived social acceptance ranged from 0.77 to 0.80 across the years.

Loneliness-Social Dissatisfaction To obtain children's selfreported feelings of loneliness and dissatisfaction with peer relations, the Loneliness and Social Dissatisfaction Scale designed by Asher et al. (1984) was completed by children each year from age 11 to 13 years. The scale has been found to be valid and reliable (Asher and Wheeler 1985). Cronbach's alpha ranged from 0.85 to 0.88 across the years. The scale contains 16 items and 8 filler items. To keep the scale similar to most of the other items in the questionnaire children filled out (including the Self-Perception Profile for Children), the items are scored from 1 to 4 . A total score was computed by summing the items measuring loneliness and social dissatisfaction, except the items 'I have lots of friends' and 'I don't have any friends'. We omitted these items to avoid overlap with the perceived social acceptance construct.

Clique Isolation Children's friendship choices were used as input for the identification of cliques, and for assessing clique isolation. Children were asked each year to nominate up to four friends in the classroom. The nominations were limited to classrooms, because all lessons were administered in a classroom context and classroom composition remained stable throughout the year. Children could nominate both boys and girls as their friends. Children were considered to have a reciprocated friendship when the 
nominated friend also nominated the target child as one of his/her four best friends (Bukowski and Hoza 1989). Based on these friendship nominations, the social network analysis (SNA) program Kliquefinder (Frank 1995, 1996) was used to identify clique isolation at age 11 years (grade 5), at age 12 years (grade 6), and at age 13 years (grade 7). Kliquefinder is based on a clustering algorithm to detect cohesive subgroups. The algorithm identifies cliques by maximizing an objective function, which looks at the probability that a child interacts with someone in his or her clique. The program detects cohesive cliques in terms of a concentration of interactions (i.e., friendship nominations) within the cliques relative to the extent of interactions across cliques. For more information about the Kliquefinder program, see Frank $(1995)^{1}$. Because we made a distinction in the present study between dyadic friendships and cliques, clique membership was conceptualized as having a minimum of two (un-)reciprocated friendship nominations with other members of the clique. Because the children in our sample could not nominate more than four children as their friends, we followed Ennett and Bauman (1994) and used both reciprocated and unreciprocated friendship nominations of all children within a classroom for input of the program. We weighted the friendship nominations so that reciprocated friendships represented twice the interaction of unreciprocated friendships (i.e., reciprocated friendships received a weight of 2 and unreciprocated friendships a weight of 1).

A dummy-coded variable was created each year to measure clique isolation (isolated from cliques $=1$; clique member $=0$ ). The output of the Kliquefinder program with the final clique solution showed that children with only one link to a clique were sometimes identified as clique members. Moreover, some cliques consisted of strings of (un-) reciprocated friendships where children were only connected to one other child in the clique. We decided to reclassify these children as isolates, because these children did not meet our predefined criteria of clique membership. We could not detect cliques in 3 out of 17 classrooms in grade $6(N=88)$ and in 2 out of 19 classrooms in grade 7 $(N=64)$ with the Kliquefinder program. Because it is uncertain whether Kliquefinder was unable to detect cliques in these classrooms or whether cliques truly didn't exist in these classrooms, the clique isolation status of these participants was handled as missing for the relevant time point.

\footnotetext{
${ }^{1}$ To check for convergence between Kliquefinder and other programs to identify cliques, we compared the final clique solution for children at age 11 years (i.e., grade 5) with results for the same cohort of the SNA program Negopy (Richards 1995). We found an overlap in the final clique solution of Kliquefinder and Negopy of more than $90 \%$, indicating high convergence.
}

Peer Rejection Children's sociometric status at age 11 years was assessed through peer nominations. Children were asked to nominate three children in their class whom they liked most and three children whom they liked least. Following Coie et al. (1982), the number of nominations for liked most and liked least items were totaled for each child and then standardized within each classroom. These scores were transformed into social preference (the standardized difference between the number of liked most and liked least nominations) and into social impact (the standardized sum of the liked most and liked least scores). Children were classified as rejected if their social preference score was less than -1 , their standardized liked most score was less than 0 , and their standardized liked least score was greater than 0 . A dummy coded variable $(1=$ rejected; $0=$ not rejected $)$ was created to indicate whether children belonged to the rejected category or not.

Friendlessness To measure friendlessness at age 11 years, a dummy coded variable $(1=$ having no reciprocated friendships; $0=$ having at least one reciprocated friendship) was created. We used a dichotomous variable, because friendlessness is typically defined as not having any reciprocal friendships (Bukowski and Hoza 1989). Reciprocated friendships were based on the friendship nomination procedure described above.

Sociodemographic Information The Blishen et al. (1987) Occupational Prestige Scale was used to obtain motherreported parental occupation. The scores are based on the average income and average education level associated with occupations in Canada. Scores were first averaged across the two parents and next across the four years the data were collected.

\section{Procedure}

Each spring, children spent 2 hours of classroom time answering questionnaires. After children were informed about the purpose of the study, they were assured that their answers would be held confidential and that they did not have to answer any of the questions if they did not want to. The order of the questionnaires was fixed for all children: The children answered the self-report questionnaires first, than the sociometric and friendship nomination items. Children were instructed to respond in silence and not to disclose their answers to anyone; we also used cardboard screens to insure privacy. Trained research assistants administered the questionnaires in the absence of the teachers to emphasize that participants' answers would not be revealed to the teachers. 


\section{Results}

\section{Descriptive Statistics}

Fifty-four (19\%), 34 (15\%), and 37 (16\%) children were isolated from cliques at age 11, 12, and 13 years, respectively. No sex differences were found in the number of clique isolates from age 11 to age 13 years. In the analyses, children who were isolated from cliques were contrasted with children who were members of cliques. These clique members belonged to cliques with an average size of 5.34 (range 3 to 11 ), 5.39 (range 3 to 11 ), and 5.70 (range 3 to 11 ) members from age 11 to age 13 years, respectively. Descriptive statistics of the variables included in this study are in Table 1. Bivariate correlations among the continuous self-reported variables loneliness, perceived social acceptance, and depressive symptoms are in Table 2.

The Link between Clique Isolation and Depressive Symptoms

We tested the link between clique isolation and depressive symptoms through latent growth modeling using Mplus

Table 1 Means and SDs of Loneliness, Perceived Social Acceptance, Depression, and the Percentage of Children who are Isolated from Cliques, are Rejected, and Have No Dyadic Friends

\begin{tabular}{|c|c|c|c|c|}
\hline & \multicolumn{2}{|c|}{ Boys } & \multicolumn{2}{|l|}{ Girls } \\
\hline & M & SD & M & SD \\
\hline \multicolumn{5}{|l|}{ Loneliness } \\
\hline 11 years & 7.19 & 5.93 & 7.05 & 5.91 \\
\hline 12 years & 7.61 & 6.83 & 6.06 & 3.80 \\
\hline 13 years & 7.03 & 5.85 & 5.75 & 4.32 \\
\hline \multicolumn{5}{|c|}{ Perceived Social Acceptance } \\
\hline 11 years & 2.97 & 0.60 & 2.89 & 0.62 \\
\hline 12 years & 2.96 & 0.62 & 2.94 & 0.55 \\
\hline 13 years & 2.91 & 0.55 & 2.94 & 0.55 \\
\hline \multicolumn{5}{|l|}{ Depression } \\
\hline 11 years & 8.20 & 5.91 & 9.23 & 6.85 \\
\hline \multirow[t]{2}{*}{14 years } & 9.06 & 7.11 & 11.97 & 7.44 \\
\hline & $\%$ & & $\%$ & \\
\hline \multicolumn{5}{|c|}{ Clique Isolation } \\
\hline 11 years & 21.3 & & 15.4 & \\
\hline 12 years & 17.1 & & 15.5 & \\
\hline 13 years & 17.1 & & 15.8 & \\
\hline \multicolumn{5}{|c|}{ Peer Rejection } \\
\hline 11 years & 15.7 & & 10.4 & \\
\hline \multicolumn{5}{|c|}{ No Dyadic Friends } \\
\hline 11 years & 19.7 & & 19.4 & \\
\hline
\end{tabular}

$\%=$ percentage of children who are clique members, who are rejected by peers, and who do not have dyadic friends, respectively
5.21 (Muthén and Muthén 1998-2009). All tested models controlled for sex and parental occupational prestige. Because clique isolation was measured as a binary variable (i.e., clique isolation versus clique membership), we specified a latent growth model for categorical data using an intercept and linear slope. In this model, the intercept of clique isolation represented individual differences in the probability of being isolated from cliques, whereas a significant slope parameter would indicate that with age, children's probability of clique isolation would change. The model fit was assessed through the Comparative Fit Index and Tucker Lewis Index (CFI and TLI, values $>0.90$ indicate acceptable to good fit; Bentler and Bonett 1980; Marsh et al. 2004), and the Root Mean Square Error of Approximation (RMSEA, value $\leq 0.08$; Browne and Cudeck 1993).

We started off by fitting a growth model on clique isolation probability from age 11 to 13 years. A non-significant estimate of the slope parameter of clique isolation probability $\left(B_{\text {slope }}=0.30, \mathrm{SE}=0.35, p>0.05\right)$ indicated that on average, children did not change in their probability of being isolated from cliques from age 11 to 13 years. Moreover, the nonsignificant variance of the slope estimate of clique isolation probability $\left(B_{\text {varslope }}=-0.00, \mathrm{SE}=0.04, p>0.05\right)$ indicated that there were no individual differences in the growth of clique isolation probability. We therefore excluded the slope parameter from the model. The variance of the intercept of clique isolation probability was significant $\left(\mathrm{var}_{\text {intcp }}=0.17\right.$, $\mathrm{SE}=0.07, p<0.05$ ), indicating that there were individual differences in the initial levels of clique isolation probability.

To test for associations between clique isolation and depressive symptoms, depression at age 14 years was regressed on the intercept of clique isolation probability. Depression at age 14 years was also regressed on depression at age 11 years. In this way, we tested whether individual differences in clique isolation probability from age 11 to 13 years predicted change in depression from age 11 to 14 years. Clique isolation was weakly correlated with peer rejection (Kendall's $\tau$ correlation, $r=0.20, p<0.05$ ) and moderately correlated with friendlessness (Kendall's $\tau$ correlation, $r=0.41, p<0.05$ ). We allowed these links between clique isolation and age 11 peer rejection and friendlessness in the model. Moreover, we also allowed paths from age 11 peer rejection and friendlessness to age 14 depression in the model to test whether clique isolation had a unique link with depression above and beyond possible links with rejection and friendlessness. We found a significant link between peer rejection and depression at age $14(\beta=0.19, p<0.01)$, but not between friendlessness and depression at age $14(\beta=0.05, p>0.05)$. The results of the test of this model are in Fig. 1. The model had a good fit to the data $(\mathrm{CFI}=0.97, \mathrm{TLI}=0.97, \mathrm{RMSEA}=0.02)$. We found that a higher probability of being isolated from cliques from 
Table 2 Bivariate Correlations among Self-reported Depression, Loneliness, and Perceived Social Acceptance

\begin{tabular}{|c|c|c|c|c|c|c|c|c|c|}
\hline & & 1. & 2. & 3. & 4. & 5. & 6. & 7. & 8 \\
\hline 1. & Depression 11 & - & & & & & & & \\
\hline 2. & Depression 14 & $0.35 * *$ & - & & & & & & \\
\hline 3. & Loneliness 11 & $0.57 * *$ & $0.28^{* *}$ & - & & & & & \\
\hline 4. & Loneliness 12 & $0.31 * *$ & $0.21 * *$ & $0.37 * *$ & - & & & & \\
\hline 5. & Loneliness 13 & $0.29 * *$ & $0.24 * *$ & $0.36 * *$ & $0.56 * *$ & - & & & \\
\hline 6. & Perc soc accept 11 & $-0.52 * *$ & $-0.15 * *$ & $-0.68 * *$ & $-0.37 * *$ & $-0.34 * *$ & - & & \\
\hline 7. & Perc soc accept 12 & $-0.33 * *$ & $-0.17 * *$ & $-0.40 * *$ & $-0.63 * *$ & $-0.43 * *$ & $0.53 * *$ & - & \\
\hline 8. & Perc soc accept 13 & $-0.23 * *$ & $-0.23 * *$ & $-0.27 * *$ & $-0.39 * *$ & $-0.64 * *$ & $0.40 * *$ & $0.51^{* *}$ & - \\
\hline
\end{tabular}

$* * p<0.01$.

Perc soc accept $=$ Perceived social acceptance

age 11 to 13 years was uniquely and significantly related to an increase in depressive symptoms from age 11 to 14 years $(B=$ $3.56, \mathrm{SE}=1.40, \beta=0.33, p<0.05$ ).

We then tested for sex differences in the observed association between clique isolation probability and depressive symptom. A two-group model was fitted, in which boys were compared with girls. The results showed no significant differences between boys and girls in the association between clique isolation probability from age 11 to 13 years, and depressive symptoms at age 14 years: Wald $(1)=0.02, p=0.88$.

As described, cliques could not be detected in 5 classrooms, and clique isolation status of the children in these classrooms was therefore handled as missing. However, possibly none of the children in these classrooms were part of a clique. We retested the model assuming that each of these children were clique isolates, making 72 and 53 of the children isolated from cliques at age 12 and 13 years, respectively. Our results remained the same. The link between clique isolation and depressive symptoms at age 14 years: $B=4.06, \mathrm{SE}=1.50, \beta=0.35, p<0.01$.

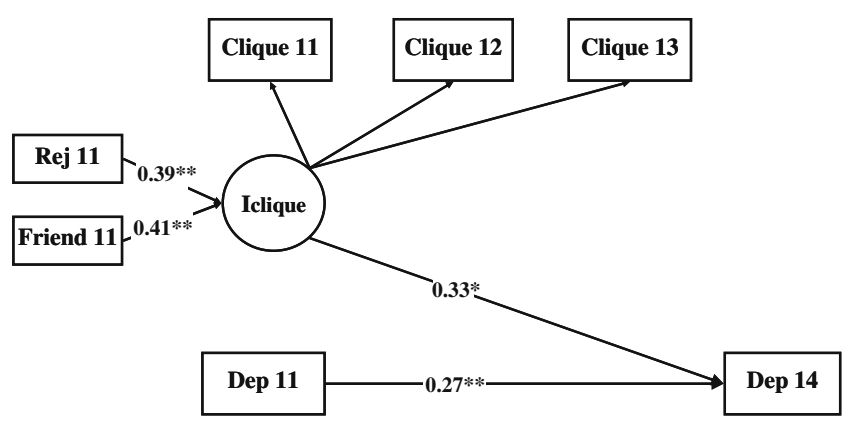

Fig. 1 Model testing the associations between clique isolation probability and depressive symptoms. Estimates were controlled for sex, parental occupational prestige, age 11 years peer rejection and friendlessness. $\mathrm{I}_{\text {clique }}=$ Intercept of Clique Isolation Probability, Dep = Depressive symptoms, Rej $=$ Peer Rejection, Friend $=$ Friendlessness. ${ }^{*} p<0.05, * * p<0.01$
Loneliness and Perceived Social Acceptance as Mediators of the Link between Clique Isolation and Depressive Symptoms

Latent factor scores for loneliness and perceived social acceptance were computed, in which the age 12 and 13 year observed scores were used as indicators of the latent factor scores. We specified two separate mediation models, which are depicted in Fig. 2 (loneliness) and Fig. 3 (perceived social acceptance). In the mediation models, the effect of clique isolation on loneliness and perceived social acceptance was estimated by regressing age 12-13 loneliness and perceived social acceptance on the intercept of clique isolation probability. To test whether the link between clique isolation and depressive symptoms was mediated by loneliness and/or perceived social acceptance, we regressed age 14 depressive symptoms on loneliness, perceived social acceptance and on the intercept of clique isolation probability. Depressive symptoms at age 14 years were controlled for depressive symptoms at age 11 years. Loneliness and perceived social acceptance at age 12-13 years were also controlled for their values at age 11 years. Moreover, the intercept of clique isolation probability was controlled for peer rejection and friendlessness at age 11 years. Finally, the significance of the indirect effect of clique isolation on depressive symptoms via loneliness and perceived social acceptance was tested (MacKinnon et al. 2004).

In the model testing loneliness as the mediator (see Fig. 2), we found a significant path from the intercept of clique isolation probability to loneliness $(B=4.02, \mathrm{SE}=1.30, \beta=$ $0.47, p<0.01)$, and a significant path from loneliness to depressive symptoms $(B=0.41, \mathrm{SE}=0.15, \beta=0.25, p<0.01)$. A test of the significance of the indirect path showed that the path of clique isolation probability on depressive symptoms via loneliness was indeed significant $(B=1.67, \mathrm{SE}=0.78, \beta=$ $0.11, p<0.05)$. Moreover, the direct path from clique isolation probability to depressive symptoms was no longer significant after loneliness was included in the model $(B=$ 


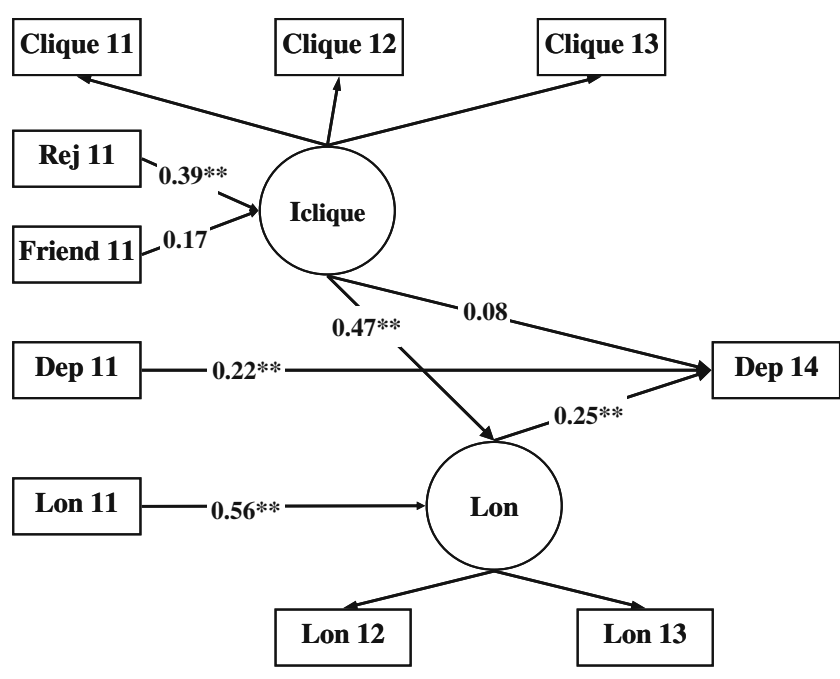

Fig. 2 Mediation model testing the associations between clique isolation, depressive symptoms, and loneliness. Estimates were controlled for sex, parental occupational prestige, age 11 years peer rejection and friendlessness. $\mathrm{I}_{\text {clique }}=$ Intercept of Clique Isolation Probability, Dep $=$ Depressive symptoms, Lon $=$ Loneliness, Rej $=$ Peer Rejection, Friend $=$ Friendlessness. ${ }^{* *} p<0.01$

1.22, $\mathrm{SE}=1.94, \beta=0.08, p=0.53$ ), suggesting that the association between clique isolation probability and depressive symptoms was mediated by loneliness (Baron and Kenny 1986; Mackinnon et al. 2007). As stated, occasional missing data were handled through FIML estimation. To test whether missing data impacted these final model results we re-estimated the model for children with only compete data $(n=269)$. The mediation path via loneliness remained significant, $B=1.54, \mathrm{SE}=0.75, \beta=0.11, p<0.05$ )

In the model testing perceived social acceptance as the mediator (see Fig. 3), we found a significant path from clique isolation probability to perceived social acceptance $(B=-0.32, \mathrm{SE}=0.10, \beta=-0.43, p<0.01)$. However, the path from perceived social acceptance to depressive symptoms was not significant $(B=0.88, \mathrm{SE}=1.28, \beta=$ $0.06, p=0.99)$. Moreover, the path from clique isolation probability to depressive symptoms remained significant after including perceived social acceptance $(B=3.71, \mathrm{SE}=$ $1.61, \beta=0.34, p<0.01)$, suggesting that the link between clique isolation and depressive symptoms was not mediated by perceived social acceptance.

To test for possible sex differences in the observed associations, we re-specified the above models as twogroup models, in which boys were compared with girls. We found that the association between clique isolation probability and loneliness was not different for boys and girls (Wald $(1)=2.61, p>0.05$ ), nor was the association between loneliness and depressive symptoms (Wald $(1)=0.30, p>$ 0.05 ). Moreover, we found that the association between clique isolation and perceived social acceptance was different (Wald $(1)=6.15, p<0.05$; only significant in boys).
The association between perceived social acceptance and depressive symptoms was similar and non-significant in both boys and girls (Wald $(1)=1.96, p>0.05$ ).

\section{Discussion}

The objectives of the present study were (a) to investigate whether clique isolation from age 11 to 13 years is a social risk factor for subsequent depressive symptoms in early adolescence; (b) to test the potential role of loneliness and perceived social acceptance as cognitive and emotional constructs underlying the link between clique isolation and depressive symptoms; and (c) to explore possible sex differences in the association between clique isolation and depressive symptoms. Accounting for children's initial level of depressive symptoms at age 11 years and for other problematic peer experiences (i.e., age 11 peer rejection and friendlessness), the results showed that the probability of being isolated from cliques from age 11 to 13 years predicted an increase in depressive symptoms from age 11 to age 14 years. Moreover, we found that loneliness, but not perceived social acceptance mediated the association between being isolated from cliques and depressive symptoms in early adolescence. Finally, we did not find sex differences in the associations between clique isolation, loneliness, and depressive symptoms. However, we found that the association between clique isolation and perceived social acceptance was only significant for boys.

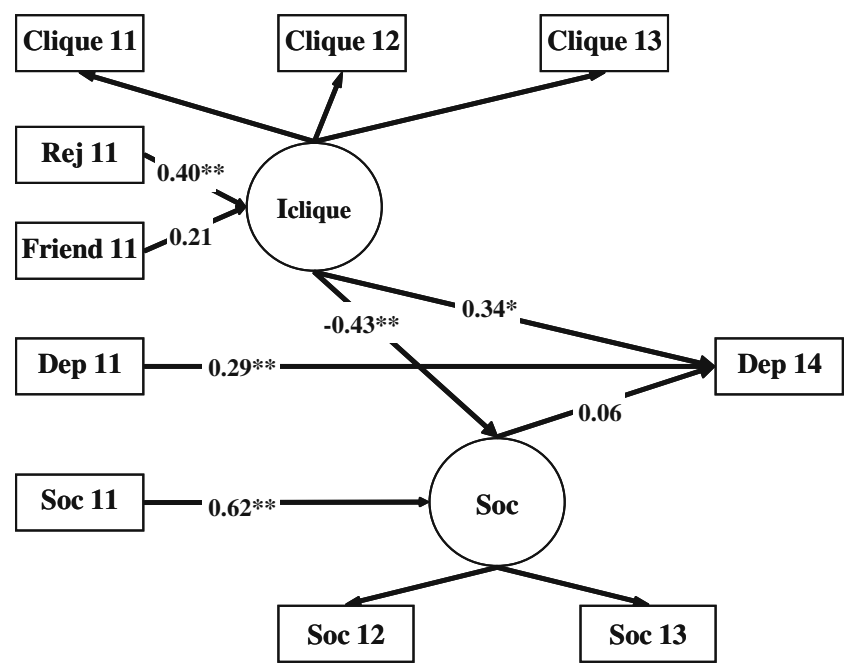

Fig. 3 Mediation model testing the associations between clique isolation, depressive symptoms, and perceived social acceptance. Estimates were controlled for sex, parental occupational prestige, age 11 years peer rejection and friendlessness. $I_{\text {clique }}=$ Intercept of Clique Isolation Probability, Dep $=$ Depressive symptoms, Soc $=$ Perceived Social Acceptance, Rej $=$ Peer Rejection, Friend $=$ Friendlessness. ${ }^{*} p<0.05,{ }^{* *} p<0.01$ 
The results found in the present study are consistent with previous research on problematic peer experiences as risk factors for the development of depression in childhood and adolescence (Birmaher et al. 1996; Boivin et al. 1994, 1995; Brendgen et al. 2005; Ge et al. 1994; Ladd and Troop-Gordon 2003; La Greca and Moore Harrison 2005; Nangle et al. 2003; Pedersen et al. 2007). Moreover, our findings provide support for the notion that multiple levels of peer relations should be investigated as predictors of depressive symptoms in adolescence (La Greca and Moore Harrison 2005). That is, the findings from the present study showed that being isolated from cliques is uniquely associated with subsequent depressive symptoms even when other aspects of problematic peer relations (i.e., peer rejection and friendlessness) were taken into account. Therefore, future studies on the role of peer relations in the development of depression could benefit from taking clique isolation into account, besides other important risk factors from the peer relations domain such as peer rejection and friendlessness.

Our findings suggest that loneliness explains the link between clique isolation and depressive symptoms in early adolescence. These results are in line with previous studies indicating that loneliness mediates the association between different types of peer difficulties (popularity, friendship quantity and friendship quality in the Nangle et al. 2003 study; social withdrawal, peer rejection and victimization in the Boivin et al. 1995 study; clique isolation in the current study) and depressed mood (Boivin et al. 1995; Nangle et al. 2003). As for experiences of peer rejection and being friendless, being isolated from cliques seems to be associated with subsequent depressive symptoms because children internalize these negative social experiences into feelings of loneliness. Thus, the results of the present study support the notion that negative group level experiences (i.e., in the present paper conceptualized as being isolated from cliques) contribute to children's feelings of loneliness and, ultimately, to depressive symptoms (Brown and Lohr 1987; Hoza et al. 2000).

Whereas loneliness mediated the link between clique isolation and depressive symptoms, no such mediation effect was found for perceived social acceptance. Our finding that the probability of being isolated from cliques from age 11 to 13 years were associated with low perceived social acceptance seems to support the theory that children's self-esteem regarding their social competencies is based on their actual experiences in the peer group (Cole 1991; Harter et al. 1996; Mead 1934). However, perceived social acceptance did not predict depressive symptoms at age 14 years above and beyond the association between clique isolation and depressive symptoms. The combined findings of mediation by loneliness but not by low perceived social acceptance seem to suggest that the acute awareness of being isolated from cliques - rather than the more indirect process of developing negative self-schemata concerning one's own role in this specific negative experience-underlies the association between clique isolation and subsequent depressive feelings. We do have to note, though, that these findings may not be true for all internalizing problems experienced by young adolescents. For instance, social anxiety is found to be related to problematic peer relations, perceived social acceptance and loneliness (e.g., La Greca and Moore Harrison 2005). The link between clique isolation and social anxiety (instead of depressive symptoms) could well be mediated by not only loneliness but also by perceived social acceptance. Consequently, future studies could benefit from taking other internalizing problems besides depressive symptoms, such as social anxiety, into account.

Although several studies focusing on adolescence have found that boys are more often isolated from cliques than girls (Cohen 1977; Urberg et al. 1995), in the present study, which focused on clique membership in late childhood, we did not find sex differences in clique membership status. This discrepancy in findings may have been the result of the difference in the investigated age periods. In adolescence, the concept of friendship changes and the emphasis on intimacy increases (Sullivan 1953). Because intimacy is believed to play a larger role among girls than among boys (Maccoby 1998), sex differences in clique membership may become apparent particularly in adolescence when intimacy is a more important friendship goal. We did find, however, that the association between clique isolation and perceived social acceptance was only true for boys and not for girls. More emphasis on group-oriented goals such as shared interests and activities in boys' friendships (Maccoby 1998) could explain why perceived social acceptance is more strongly related to problematic peer experiences at the group level for boys than for girls.

In line with earlier findings about the association between problematic peer relations and depression in childhood and adolescence (Ladd and Troop-Gordon 2003; La Greca and Moore Harrison 2005; Pedersen et al. 2007), we did not find sex differences in the association between clique isolation and subsequent depressive symptoms and also not in the mediating role of loneliness and social self perception. Thus, clique isolation seems to be a social risk factor for depressive symptoms in early adolescence for both boys and girls.

\section{Limitations and Conclusions}

The present study is not without limitations. First, our results do not imply causal links between clique isolation and internalizing problems. Although we used a longitudinal design and controlled for initial levels of depression, 
loneliness, and perceived social acceptance at age 11 years, it cannot be ruled out that other constructs not measured in the present study, including other forms of internalizing problems such as anxiety, underlie the observed associations between clique isolation and depressive symptoms. Also, the possibility exists that clique isolation did not cause the internalizing problems measured in the present study (i.e., loneliness, perceived social acceptance and depressive symptoms), but that clique isolation was the result of internalizing problems. According to the interpersonal theory of depression (Coyne 1976), problematic social relationships may be consequences of depression and may at the same time worsen a person's depressive feelings. Indeed, this may suggest a transactional relationship between clique isolation and internalizing problems. Future studies could further test this potential transactionality by not only regarding peer problems as risk factors for the escalation of depressive symptoms, but also as consequences of internalizing problems.

The second limitation concerns the time frame of the present study. We focused on depressive symptoms in early adolescence (i.e., at age 14 years). Evidence has been found, however, for a sharp increase in depression and an increase in sex differences in depression between age 15 and 18 years (Hankin et al. 1998). This may suggest that, rather than early adolescence, middle-to-late adolescence is an essential period for studying depression and related risk- and protective factors. In contrast to these findings, however, Brendgen et al. (2005) and Costello et al. (2008) recently showed that the increase in depressive symptoms may already begin during early adolescence. Moreover, young adolescents who display depressive symptoms are at great risk for developing major depressive episodes later on (Pine et al. 1999). Therefore, depressive symptoms in children and young adolescents warrant further attention by research.

Third, in our assessment of clique membership and clique isolation, children could nominate up to four friends. Although we included both reciprocated and unreciprocated friendship nominations for the identification of cliques, the limited friendship nominations could have resulted in smaller cliques and more clique isolates compared to the use of unlimited friendship nominations. Nonetheless, the average clique size in the present study (between 5 and 6 members) and the average number of clique isolates (between $16 \%$ and 19\%) is in accordance with findings from studies that used unlimited friendship nominations or that used other methods to identify cliques (e.g., Espelage et al. 2003; Kindermann 1993; Urberg et al. 1995).

A fourth limitation involves the fact that loneliness, perceived social acceptance, and depressive symptoms were all measured with self-reports. That is, we cannot rule out that some of our findings may have resulted from sharedmethod variance. Information from other raters, such as teachers and parents could rule out this possibility. However, teachers and parents may not have full access to children's internal experiences and may therefore underreport the severity and frequency of children's internalizing problems (Crowley et al. 1992).

Finally, this study used an ethnically homogeneous sample of young adolescents of French Canadian background. Studies using a more ethnically diverse sample are needed to increase the generalizability of the present findings.

Despite these limitations, the results from the present study offer new insights in the link between problematic peer relations and depressive symptoms that may have important implications for research and prevention. That is, the finding that clique isolation predicted an increase in depressive symptoms indicates that viewing peer relations from a group perspective contributes significantly to the existing knowledge about problematic peer experiences as social risk factors for depression in early adolescence. In addition, the finding that loneliness but not perceived social acceptance mediated the link between clique isolation and depressive symptoms provides insight into underlying cognitive-emotional constructs, which may be helpful in preventing depressive symptoms in early adolescence. Specifically, prevention efforts could benefit from viewing internalizing problems in early adolescence as phenomena that occur within a group context. Moreover, a focus on the development of feelings of loneliness in children who are isolated from cliques may be a central component in preventing subsequent depressive symptoms in these children. Indeed, because early adolescents with depressive symptoms are at great risk for developing major depressive disorder, the identification of elements that can be used in preventing depressive symptoms is an important goal.

Acknowledgement This research was made possible by grants to the last author from the Social Sciences and Humanities Research Council of Canada and the Fonds Québécois de la Recherche sur la Société et la Culture. We wish to thank the participating families, and the authorities and directors as well as the teachers of the participating schools. We also thank Jacqueline Langlois and Ken Frank for their assistance in the preparation of the data.

Open Access This article is distributed under the terms of the Creative Commons Attribution Noncommercial License which permits any noncommercial use, distribution, and reproduction in any medium, provided the original author(s) and source are credited.

\section{References}

Abramson, L. Y., Alloy, L. B., \& Metalsky, G. I. (1989). Hopelessness depression: a theory-based subtype of depression. Psychological Review, 96, 358-372.

Adler, P. A., \& Adler, P. (1998). Peer power: Preadolescent culture and identity. New Brunswick: Rutgers University Press. 
Allgood-Merten, B., Lewinsohn, P. M., \& Hops, H. (1990). Sexdifferences and adolescent depression. Journal of Abnormal Psychology, 99, 55-63.

Asher, S. R., \& Wheeler, V. A. (1985). Children's loneliness: a comparison of rejected and neglected peer status. Journal of Consulting and Clinical Psychology, 53, 500-505.

Asher, S. R., Hymel, S., \& Renshaw, P. D. (1984). Loneliness in children. Child Development, 55, 1456-1464.

Bagwell, C. L., Coie, J. D., Terry, R. A., \& Lochman, J. E. (2000). Peer clique participation and social status in preadolescence. Merrill-Palmer Quarterly, 46, 280-305.

Baron, R. M., \& Kenny, D. A. (1986). The moderator-mediator variable distinction in social psychological research: conceptual, strategic and statistical considerations. Journal of Personality and Social Psychology, 51, 1173-1182.

Beck, A. T. (1987). Cognitive models of depression. Journal of Cognitive Psychotherapy, 1, 5-37.

Bentler, P. M., \& Bonett, D. G. (1980). Significance tests and goodness of- fit in the analysis of covariance structures. Psychological Bulletin, 88, 588-606.

Birmaher, B., Ryan, N. D., Williamson, D. E., Brent, D. A., Kaufman, J., Dahl, R. E., et al. (1996). Childhood and adolescent depression: a review of the past 10 years. Part 1. Journal of the American Academy of Child and Adolescent Psychiatry, 35, $1575-1583$.

Blishen, B. R., Carroll, W. K., \& Moore, C. (1987). The 1981 socioeconomic index for occupations in Canada. Canadian Review of Sociology and Anthropology, 24, 465-488.

Boivin, M., Vitaro, F., \& Gagnon, C. (1992). A reassessment of the selfperception profile for children: factor structure, reliability, and convergent validity of a French version among 2nd through 6th grade children. International Journal of Behavioral Development, 15, 275-290.

Boivin, M., Poulin, F., \& Vitaro, F. (1994). Depressed mood and peer rejection in childhood. Development and Psychopathology, 6, 483-498.

Boivin, M., Hymel, S., \& Bukowski, W. M. (1995). The roles of social withdrawal, peer rejection, and victimization by peers in predicting loneliness and depressed mood in childhood. Development and Psychopathology, 7, 765-785.

Brage, D., \& Meredith, W. (1994). A causal model of adolescent depression. Journal of Psychology, 128, 455-468.

Brendgen, M., Wanner, B., Morin, A. J. S., \& Vitaro, F. (2005). Relations with parents and with peers, temperament, and trajectories of depressed mood during early adolescence. Journal of Abnormal Child Psychology, 33, 579-594.

Brown, B. B., \& Lohr, M. J. (1987). Peer-group affiliation and adolescent self-esteem: an integration of ego-identity and symbolic-interaction theories. Journal of Personality and Social Psychology, 52, 47-55.

Browne, M. W., \& Cudeck, R. (1993). Alternative ways of assessing model fit. In K. A. Bollen \& J. S. Long (Eds.), Testing structural equation models (pp. 136-162). Newbury Park: SAGE.

Buhrmester, D. (1990). Intimacy of friendship, interpersonal competence, and adjustment during preadolescence and adolescence. Child Development, 61, 1101-1111.

Buhrmester, D., \& Furman, W. (1987). The development of companionship and intimacy. Child Development, 58, 11011113.

Bukowski, W., \& Hoza, B. (1989). Popularity and friendship: Issues in theory, measurement, and outcome. In T. J. Berndt \& G. W. Ladd (Eds.), Peer relationships in child development (pp. 15-45). New York: Wiley.

Cohen, J. M. (1977). Sources of peer group homogeneity. Sociology of Education, 50, 227-241.
Coie, J. D., Dodge, K. A., \& Coppotelli, H. (1982). Dimensions and types of social-status: a cross-age perspective. Developmental Psychology, 18, 557-570.

Cole, D. A. (1991). Preliminary support for a competency-based model of depression in children. Journal of Abnormal Psychology, 100, 181-190.

Costello, D. M., Swendsen, J., Rose, J. S., \& Dierker, L. C. (2008). Risk and protective factors associated with trajectories of depressed mood from adolescence to early adulthood. Journal of Consulting and Clinical Psychology, 76, 173-183.

Coyne, J. C. (1976). Toward an interactional description of depression. Psychiatry-Interpersonal and Biological Processes, 39, 28-40.

Crowley, S. L., Worchel, F. F., \& Ash, M. J. (1992). Self-report, peerreport, and teacher-report measures of childhood depression: an analysis by item. Journal of Personality Assessment, 59, 189203.

Ennett, S. T., \& Bauman, K. E. (1994). The contribution of influence and selection to adolescent peer group homogeneity: the case of adolescent cigarette-smoking. Journal of Personality and Social Psychology, 67, 653-663.

Espelage, D. L., Holt, M. K., \& Henkel, R. R. (2003). Examination of peer-group contextual effects on aggression during early adolescence. Child Development, 74, 205-220.

Frank, K. A. (1995). Identifying cohesive subgroups. Social Networks, $17,27-56$.

Frank, K. A. (1996). Mapping interactions within and between cohesive subgroups. Social Networks, 18, 93-119.

Fontaine, K. G., Yang, C., Salzer Burks, V., Dodge, K. A., Price, J. M., Pettit, G. S., et al. (2009). Loneliness as a partial mediator of the relation between low social preference in childhood and anxious/depressed symptoms in adolescence. Development and Psychopathology, 21, 79-491.

Fundulis, T., Berney, T. P., Kolvin, O., Famuyiva, O. O., Barrett, T., Bhate, S., et al. (1991). Reliability and validity of two rating scales in the assessment of childhood depression. British Journal of Psychiatry, 159, 36-40.

Ge, X. J., Lorenz, F. O., Conger, R. D., Elder, G. H., \& Simons, R. L. (1994). Trajectories of stressful life events and depressive symptoms during adolescence. Developmental Psychology, 30, 467-483.

Hankin, B. L., Abramson, L. Y., Moffitt, T. E., Silva, P. A., McGee, R., \& Angell, K. E. (1998). Development of depression from preadolescence to young adulthood: emerging gender differences in a 10-year longitudinal study. Journal of Abnormal Psychology, $107,128-140$.

Harter, S. (1982). The perceived competence scale for children. Child Development, 53, 87-97.

Harter, S. (1985). Manual for the self-perception profile for children. Denver: University of Denver.

Harter, S., Stocker, C., \& Robinson, N. S. (1996). The perceived directionality of the link between approval and self-worth: the liabilities of a looking glass self-orientation among young adolescents. Journal of Research on Adolescence, 6, 285-308.

Henrich, C. C., Kuperminc, G. P., Sack, A., Blatt, S. J., \& Leadbeater, B. J. (2000). Characteristics and homogeneity of early adolescent friendship groups: a comparison of male and female clique and nonclique members. Applied Developmental Science, 4, 15-26.

Hoza, B., Bukowski, W. M., \& Beery, S. (2000). Assessing peer network and dyadic loneliness. Journal of Clinical Child Psychology, 29, 119-128.

Joiner, T. E. (1997). Shyness and low social support as interactive diatheses, with loneliness as mediator: testing an interpersonalpersonality view of vulnerability to depressive symptoms. Journal of Abnormal Psychology, 106, 386-394.

Joiner, T. E., Jr., Lewinsohn, P. M., \& Seeley, J. R. (2002). The core of loneliness: lack of pleasurable engagement -more so than painful 
disconnection-predicts social impairment, depression onset, recovery from depressive disorders among adolescents. Journal of Personality Assessment, 79, 472-491.

Kindermann, T. A. (1993). Natural peer groups as contexts for individual development: the case of children's motivation in school. Developmental Psychology, 29, 970-977.

Kindermann, T. A. (2007). Effects of naturally existing peer groups on changes in academic engagement in a cohort of sixth graders. Child Development, 78, 1186-1203.

Kindermann, T., \& Gest, S. D. (2009). Assessment of the peer group: Identifying naturally occurring social networks and capturing their effects. In K. Rubin, W. Bukowski, \& B. Laursen (Eds.), Handbook of peer interactions, relationships and groups. New York: Guilford Press.

Kovacs, M. (1992). Children's depression inventory (CDI) manual. Toronto: Multi-Health Systems.

Kovacs, M., \& Beck, A. T. (1978). Maladaptive cognitive structures in depression. American Journal of Psychiatry, 135, 525-533.

Ladd, G. W., \& Troop-Gordon, W. (2003). The role of chronic peer difficulties in the development of children's psychological adjustment problems. Child Development, 74, 1344-1367.

La Greca, A. M., \& Moore Harrison, H. (2005). Adolescent peer relations, friendships, and romantic relationships: do they predict social anxiety and depression? Journal of Clinical Child and Adolescent Psychology, 34, 49-61.

Maccoby, E. E. (1998). The two sexes: Growing up apart, coming together. Cambridge: Harvard University Press.

MacKinnon, D. P., Lockwood, C. M., \& Williams, J. (2004). Confidence limits for the indirect effect: distribution of the product and resampling methods. Multivariate behavioral research, 39, 99-128.

MacKinnon, D. P., Fairchild, A. J., \& Fritz, M. S. (2007). Mediation analysis. Annual Review of Psychology, 58, 593-614.

MacPhee, A. R., \& Andrews, J. J. W. (2006). Risk factors for depression in early adolescence. Adolescence, 41, 435-466.

Marsh, H. W., Hau, K., \& Wen, Z. (2004). In search of golden rules: comment on hypothesis-testing approaches to setting cutoff values for fit indexes and danger in overgeneralizing $\mathrm{Hu}$ and Bentler's (1999) findings. Structural Equation Modeling, 11, $320-341$.

Mead, G. H. (1934). Mind, self, and society from the standpoint of a social behaviorist. Chicago: University of Chicago Press.

Muthén, L. K., \& Muthén, B. O. (1998-2009). Mplus User's guide (5th ed.). Los Angeles: Muthén \& Muthén.

Nangle, D. W., Erdley, C. A., Newman, J. E., Mason, C. A., \& Carpenter, E. M. (2003). Popularity, friendship quantity, and friendship quality: interactive influences on children's loneliness and depression. Journal of Clinical Child and Adolescent Psychology, 32, 546-555.

Orth, U., Robins, R. W., \& Roberts, B. W. (2008). Low self-esteem prospectively predicts depression in adolescence and young adulthood. Journal of Personality and Social Psychology, 95, 695-708.

Parker, J. G., \& Asher, S. R. (1993). Friendship and friendship quality in middle childhood: links with peer group acceptance and feelings of loneliness and social dissatisfaction. Developmental Psychology, 29, 611-621.

Parker, J. G., \& Gottman, J. (1989). Social and emotional development in a relational context: Friendship interaction from early childhood to adolescence. In T. J. Berndt \& G. W. Ladd (Eds.), Peer relationships in child development (pp. 95-131). New York: Wiley.

Pedersen, S., Vitaro, F., Barker, E. D., \& Borge, A. I. H. (2007). The timing of middle-childhood peer rejection and friendship: linking early behavior to early-adolescent adjustment. Child Development, 78, 1037-1051.

Pelkonen, M., Marttunen, M., Kaprio, J., Huurre, T., \& Aro, H. (2008). Adolescent risk factors for episodic and persistent depression in adulthood. A 16-year prospective follow-up study of adolescents. Journal of Affective Disorders, 106, 123-131.

Petersen, A. C., Compas, B. E., Brooksgunn, J., Stemmler, M., Ey, S., \& Grant, K. E. (1993). Depression in adolescence. American Psychologist, 48, 155-168.

Pine, D. S., Cohen, E., Cohen, P., \& Brook, J. (1999). Adolescent depressive symptoms as predictors of adult depression: moodiness or mood disorder? American Journal of Psychiatry, 156, 133-135.

Richards, W. D. (1995). NEGOPY 430 Manual and User's Guide. Burnaby: School of Communications, Simon Fraser University.

Rubin, Z. (1980). Children's friendships. Cambridge, Massachusetts: Harvard University Press.

Rubin, K. H., Burgess, K. B., Kennedy, A. E., \& Stewart, S. L. (2003). Social withdrawal in childhood. In E. J. Mash \& R. A. Barkley (Eds.), Child psychopathology (2nd ed., pp. 372-406). New York: Guilford Press.

Rudolph, K. D., Flynn, M., \& Abaied, J. L. (2008). A developmental perspective on interpersonal theories of youth depression. In J. R. Z. Abela \& B. L. Hankin (Eds.), Handbook of depression in children and adolescents (pp. 79-102). New York: Guilford Press.

Shrum, W., \& Cheek, N. H. (1987). Social-structure during the school years: onset of the degrouping process. American Sociological Review, 52, 218-223.

Sullivan, H. S. (1953). The interpersonal theory of psychiatry. New York: Norton.

Urberg, K. A., Degirmencioglu, S. M., Tolson, J. M., \& Hallidayscher, K. (1995). The structure of adolescent peer networks. Developmental Psychology, 31, 540-547.

Wasserman, S., \& Faust, K. (1994). Social network analysis: Methods and applications. Cambridge University Press.

Wentzel, K. R., \& Caldwell, K. (1997). Friendships, peer acceptance, and group membership: relations to academic achievement in middle school. Child Development, 68, 1198-1209. 